\title{
UTILIZACIÓN DE BIOENSAYOS PARA LA DETERMINACIÓN DE CONTAMINACIÓN EN AGUA DE RIEGO EN LA CUENCA DEL RÍO GRANOBLES
}

\author{
* Bio. Rocío Contero \\ * Ing. Orlando Felicita
}

\begin{abstract}
ANTECEDENTES
El uso de los bioensayos como indicadores del grado de afectación de una sustancia química en organismos vivos, ha sido manejado bajo condiciones controladas de laboratorio desde hace mucho tiempo; se ha trabajado con éxito en varios países latinoamericanos (Colombia, Chile, Argentina, Cuba y otros), especialmente utilizando cebolla de bulbo (Allium cepa), lechuga (Lactuca sativa), pulga de agua (Daphnia magna), algas (Hidra attenuata), arroz (Oriza sativa), repollito de agua (Pistia stratiotes), larva y adultos de Aedes aegypty, entre las principales.
\end{abstract}

El Centro de Estudios y Asesoría en Salud (CEAS) Centro de Estudios Ambientales, con el auspicio del CIID de Canadá, ejecuta el proyecto ECO SALUD Ruptura del Ecosistema Floricultor e Impacto en la Salud Humana en Cayambe; Abordaje Participativo Hacia un Ecosistema Saludable (Ecuador 100661001), en la cuenca del río Granobles desde enero de 2002.

En septiembre de 2003 se firma el convenio de cooperación entre el CEAS (PROYECTO ECOSALUD), UNIVERSIDAD POLITECNICA SALESIANA (CARRERA DE INGENIERIA EN PRODUCCIÓN AGROPECUARIA), CASA

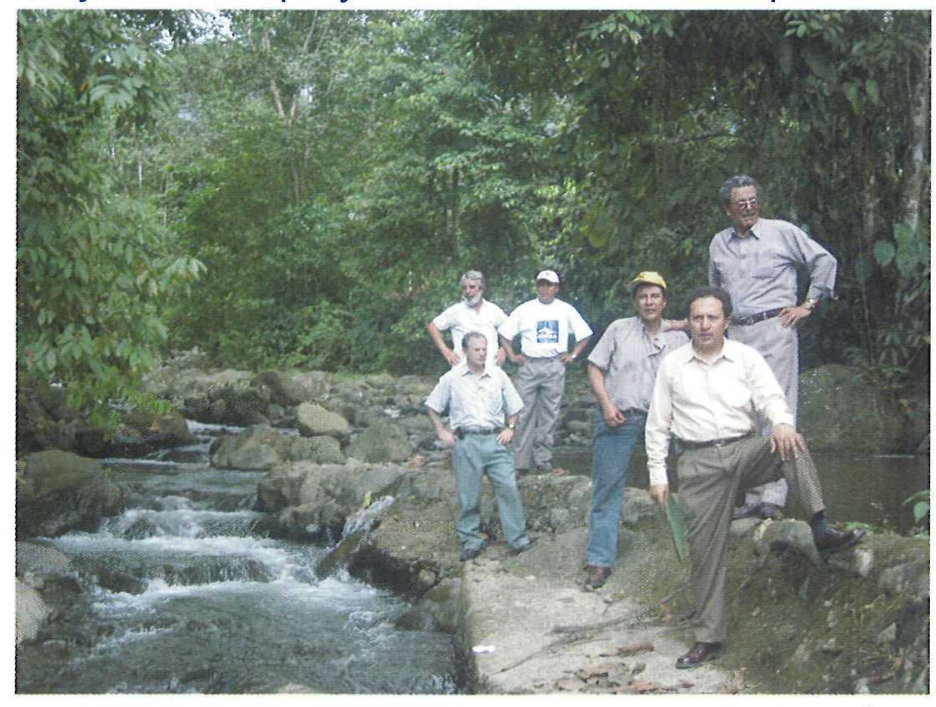
CAMPESINA Y LA UNIDAD EDUCATIVA DOMINGO SAVIO, que consiste en el montaje y puesta en marcha de un laboratorio de bioensayos para evaluar la toxicidad de agua en la cuenca del río Granobles (cantón Cayambe y Tabacundo).

A partir de la firma del convenio se trabaja en la identificación y ubicación de los diferentes sitios de muestreo en fuentes de agua, canales de riego, puntos de descarga de aguas residuales, cursos de ríos y quebradas, específicamente en las poblaciones de La Chimba, Agualongo, La Josefina, Cananvalle, con proyección a otros lugares.

En enero de 2004 se empezó a trabajar en el laboratorio de bioensayos, con determinación de especies, condiciones ambientales, pruebas preliminares de bioensayos a fin de definir materiales, métodos y procedimientos, entre otras actividades. Actualmente se han definido estos aspectos con los que se espera obtener resultados rápidos, claros y confiables. 


\section{INTRODUCCION}

Los continuos avances en la agricultura, desde la rotación de cosechas, pasando por el uso de residuos de cultivo, cenizas de madera, hasta llegar a plaguicidas y fertilizantes químicos ha provocado un cambio notable en el ecosistema. La zona de Cayambe y Tabacundo en los últimos tiempos han experimentado una gran proliferación de la industria florícola, que utiliza cantidades considerables de productos químicos cuyos residuos son generalmente conducidos por canales a cursos de ríos y quebradas.

El uso de pesticidas en la agricultura tradicional, la falta de servicios básicos y un crecimiento poblacional descontrolado y sin planificación en la zona, ha permitido el deterioro de ríos y quebradas, que son una importante fuente de abastecimiento de agua para la zona. Las descargas de diferentes actividades se las hace directamente a los cauces sin considerar el uso posterior del agua, especialmente por las poblaciones asentados aguas abajo que utilizan este líquido en sus diferentes actividades, lo que ha provocado problemas de salud en los moradores, deterioro del ambiente, pérdida de cosechas de productos tradicionales, inclusive abandono de las actividades agrícolas, afectando de este modo a la economía de la región.

Determinar del grado de toxicidad del agua mediante bioensayos y el conocimiento de las posibles fuentes de contaminación nos permitirá emitir criterios para establecer normas, leyes, reglamentos para el uso adecuado del recurso agua y su disposición.

\section{BIOENSAYOS}

Los bioensayos son estudios de las respuestas fisiológicas o poblacionales de organismos seleccionados que se ponen en contacto con concentraciones o proporciones crecientes de las muestras

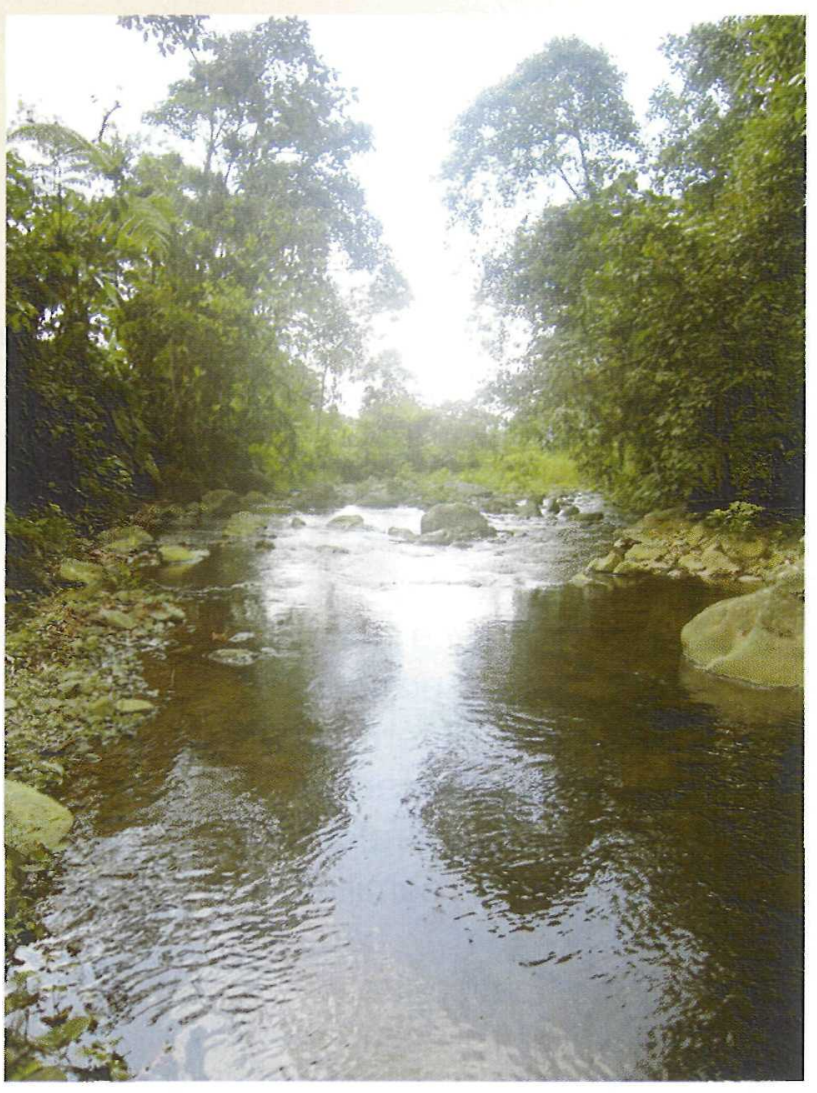
obtenidas en la zona de estudio, permiten evaluar experimentalmente el efecto de los agentes contaminantes presentes en una muestra de modo integral sobre organismos. La realización periódica de bioensayos permite detectar el aumento, disminución o mantenimiento del nivel de adversidad en un determinado ambiente. La ventaja de los bioensayos sobre las otras técnicas utilizadas es la rapidez, facilidad, bajo costo y el obtener un efecto global de los contaminantes presentes en la muestra, ya que los vertidos de los contaminantes al medio acuático suelen ser discontinuos o puntuales, escapando a los análisis químicos rutinarios; por el contrario, los organismos biológicos expresan las alteraciones que operan durante cierto tiempo en un ecosistema.

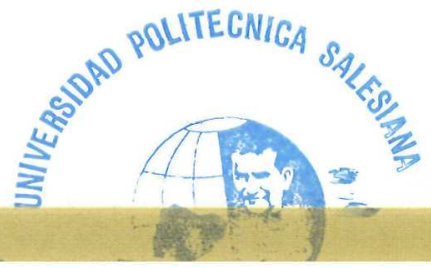




\section{OBJETIVO DEL PROYECTO}

Instalación y puesta en marcha de un laboratorio para la realización de estudios piloto en las aguas superficiales de la cuenca del río Granobles para determinar el nivel de toxicidad mediante bioensayos.

\section{MATERIALES Y MÉTODOS}

\section{Selección de especies}

Se utilizaron bulbos de cebolla (Allium cepa) de 1.25 a $2 \mathrm{~cm}$ de diámetro, cultivados en campo abierto bajo observación. Inicialmente se trabajó con cebollas del mercado, pero la evaluación de los tratamientos no fue satisfactoria, por el maltrato y el desconocimiento de la edad, variedad y las aplicaciones recibidas durante el cultivo. Los mejores resultados se obtuvieron con bulbos de 2 meses de edad de cebollas germinadas en invernadero. Los bulbos son lavados, limpiados y cortados las raicillas.

En el caso de la lechuga, es importante seleccionar la variedad comercial más utilizada en la zona, con fecha actualizada. Previa la implementación del ensayo es necesario remojar las semillas durante 2 horas. Este procedimiento permite eliminar los desinfectantes presentes normalmente en variedades comerciales.

\section{Evaluación del efecto contaminante}

Se utilizan 5 soluciones, tomando en cuenta los límites permitidos en normas para agua. Soluciones de sulfato de cobre para el caso de la cebolla y de sulfato de zinc para el caso de la lechuga. Se realizaron seis repeticiones para cada bioensayo, incluyéndose en todos los casos agua de vertiente como testigo y para control positivo, agua de la toma. Al término de la incubación, se procede a medir la longitud de las raíces de los bulbos, y de las semillas germinadas en cada disolución y de los controles. En los bulbos de cebolla y semillas de lechuga son notorias la inhibición de las

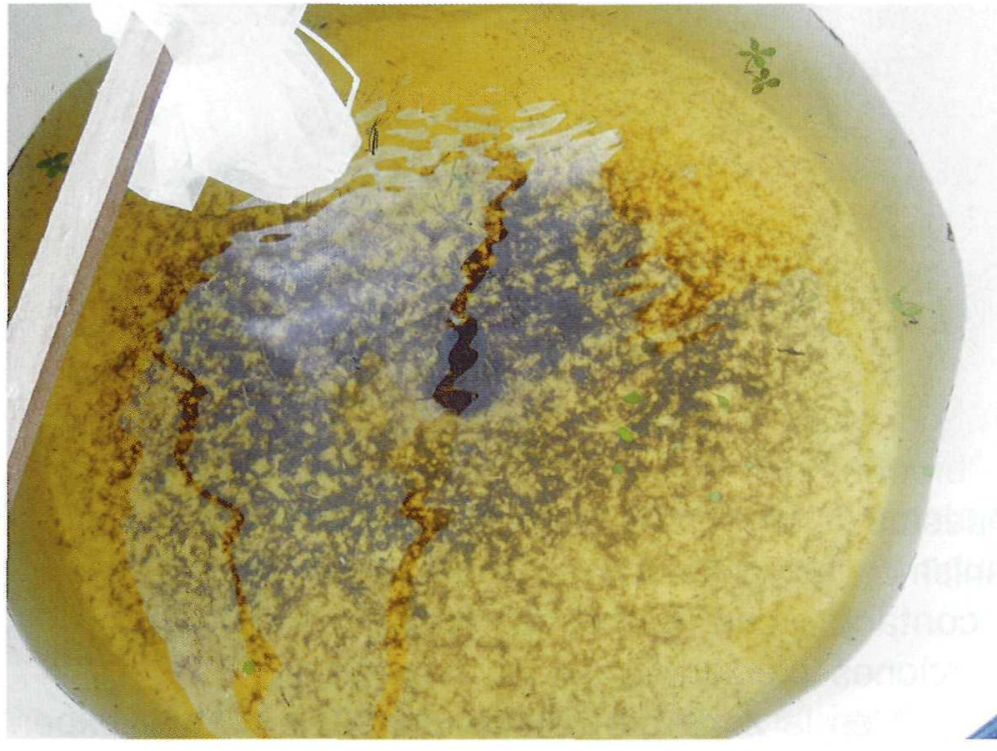
raíces a medida que aumenta la concentración del contaminante. Es importante observar semillas con crecimiento anormal (necrosis radicular o de los cotiledones, raicillas ensortijadas, etc.) o desarrollo de hongos.

\section{Preparación de soluciones}

Las soluciones se prepararon utilizando agua de vertiente y se han tomado en cuenta los límites permitidos en normas para diferentes usos de agua de acuerdo a los datos presentados a continuación. 


\begin{tabular}{|c|c|c|c|c|}
\hline SOLUCIÓN & $\begin{array}{c}\text { CuSO4 } \\
(\mathbf{M g})\end{array}$ & $\begin{array}{c}\text { AGUA } \\
(\mathbf{M I} .)\end{array}$ & $\begin{array}{c}\text { CONCENTRACIÓN } \\
\mathrm{Cu}^{+2}(\mathbf{p p m})\end{array}$ & $\begin{array}{c}\text { CONCENTRACIÓN } \\
(\%)\end{array}$ \\
\hline 1 & 1,26 & 1000 & 0,5 & 20 \\
\hline 2 & 2,51 & 1000 & 1 & 40 \\
\hline 3 & 3,77 & 1000 & 1,5 & 60 \\
\hline 4 & 5,03 & 1000 & 2 & 80 \\
\hline 5 & 6,28 & 1000 & 2,5 & 100 \\
\hline
\end{tabular}

Tabla No 1.- Datos para la preparación de soluciones de sulfato de cobre, se considera que la solución con mayor concentración corresponde al $100 \%$. ( pH promedio 7).

\begin{tabular}{|c|c|c|c|c|}
\hline SOLUCIÓN & $\begin{array}{c}\text { CuSO4 } \\
(\mathrm{Mg})\end{array}$ & $\begin{array}{c}\text { AGUA } \\
(\mathrm{MI} .)\end{array}$ & $\begin{array}{c}\text { CONCENTRACIÓN } \\
\mathrm{Zn}^{+2}(\mathrm{ppm})\end{array}$ & $\begin{array}{c}\text { CONCENTRACIÓN } \\
(\%)\end{array}$ \\
\hline 1 & 6,19 & 1000 & 2,5 & 20 \\
\hline 2 & 12,38 & 1000 & 5 & 40 \\
\hline 3 & 18,56 & 1000 & 7,5 & 60 \\
\hline 4 & 24,77 & 1000 & 10 & 80 \\
\hline 5 & 30,96 & 1000 & 12,5 & 100 \\
\hline
\end{tabular}

Tabla No 2.- Datos para la preparación de soluciones de sulfato de zinc, se considera que la solución con mayor concentración corresponde al $100 \%$. (pH promedio 7) .

Se utilizan de 15 a $20 \mathrm{ml}$ de solución de $\mathbf{C u}+2$ para el caso de la cebolla, en las diferentes concentraciones, más los controles. Para el caso de Lactuca sativa se utilizan 6-7ml de solución de $Z \mathbf{n}+2$.

\section{Descripción del método}

La investigación se está realizando en las instalaciones de la Unidad Educativa Domingo Savio, en un cuarto que mantiene una temperatura promedio de $15^{\circ}-17^{\circ} \mathrm{C}$ con una temperatura mínima de $13^{\circ} \mathrm{C}$ y máxima de $18.5^{\circ} \mathrm{C}$ y una humedad relativa promedio de $58 \%$, con una mínima de $52 \%$.

Para el caso de la cebolla se utilizaron tubos de ensayo de $10 \mathrm{ml}$ y $1.5 \mathrm{~cm}$ de diámetro, y se trabaja con luz indirecta.

En Allium cepa, las raicillas deben mantenerse siempre húmedas, este control se realiza con un intervalo mínimo de 2 horas. 
La toma de datos, el número y tamaño de raíces y el cambio de solución, se realiza diariamente y a una misma hora. El tiempo de la prueba es de 6 días.

En el caso de la lechuga, las condiciones ambientales son similares. Se siembra en cajas petri tamaño estándar, previa la colocación de papel filtro saturado con $4 \mathrm{ml}$ de solución, donde se ubican las semillas distribuidas homogéneamente. Durante el ensayo las cajas deben permanecer en un lugar oscuro, por lo que se utiliza una bolsa plástica negra para cubrirlas. Se realizan observaciones diariamente y a una misma hora, y se adiciona $2 \mathrm{ml}$ de solución a las $72 \mathrm{~h}$. El período del ensayo es de 120 horas (5 días).

\section{BIBLIOGRAFÍA}

1. CETESB. Bioensaios Microbianos Aplicados no Controle de Contaminantes Tóxicos Ambientais; Serie Didáctica, PROCOP 1991, 1-75.

2. Gustavson KE, Sonsthagen SA, Crunkilton RA, Harkin JM. Groundwater toxicity assessment using bioassay, chemical, and toxicity identification evaluation analysis. Environm Toxicol 2000;(15):421-430.

3. Montaje y Puesta en marcha de un Laboratorio de Bioensayos para evaluar la toxicidad de las aguas a nivel local. Municipalidad de Pudahuel-Santiago -Chile. [Sitio de Internet]. 2001. [Citado el 26 de octubre de 2001].[6p].Disponible en http:nmatus@hotmail.com

4. Red Internacional Watertox. [Sitio de Internet] 2000. [citado 3 de Julio del 2003]: [3p]. Disponible en URL: http://\%20www.idrc.ca/lacro/bioensayos/manual.html

5. Díaz-Baez MC, Pérez JB. Intralaboratory experience with a battery of bioassays: Colombia experience. Environm Toxicol 2000;(15):297-303

6. Castillo Luisa Eugenia, Pinnock Margaret, Martínez Eduardo. Evaluation of a battery of toxicity tests for use in the assessment of water quality in a Costa Rican Laboratory. Environm Toxicol 2000;(15):312-21.

7. Pimentel, D. 1998. Environmental and economic issues associated with pesticide use. Book of abstracts International Conference on Pesticide use in Developing Countries: Impact on Health and Environment, Costa Rica. 272 pp.

* Catedrática de Biología

** Profesor invitado del C.E.A.S. 